\title{
ОСОБЕННОСТИ УРОВНЕЙ ДВИГАТЕЛЬНОЙ АКТИВНОСТИ И КОРРЕЛЯЦИОННЫХ ВЗАИМООТНОШЕНИЙ ХАРАКТЕРИСТИК БИМАНУАЛЬНОЙ КООРДИНАЦИИ У ЛИЦ С РАЗНЫМИ ТИПАМИ КОГНИТИВНОГО СТИЛЯ ПОЛЕЗАВИСИМОСТИ- ПОЛЕНЕЗАВИСИМОСТИ
}

\author{
(С) Соколова Н.И., Ткаченко П.В., Петрова Е.В.
}

\author{
Кафедра нормальной физиологии им. профессора А.В. Завьялова \\ Курского государственного медицинского университета, Курск \\ E-mail: pwtkachenko@rambler.ru
}

\begin{abstract}
Методом суппортметрии изучены уровни бимануальной координации у мужчин и женщин, относящихся к разным типам когнитивного стиля полезависимость-поленезависимость по результатам методики включенных фигур. Сравнение средних значений показателей координации и их вариативности раскрывают возможные механизмы половых различий, связанные с подходами к решению перцептивных задач. Выявленные особенности корреляционных сопоставлений характеристик двигательной активности позволили раскрыть связи уровня восприятия и типа ориентации с особенностями реализации бимануальных движений. У мужчин связи дифференцированы по времени выполнения задания, а у женщин - по дефекту реализации трека и его сложности, что подтверждает различия в способах контроля соответствующей деятельности. При этом у женщин выявлены разные степени врабатываемости в группах с полярными типами когнитивного стиля и определяется особенностями восприятия и консолидацией эффекторных проявлений при усложнении задачи.
\end{abstract}

Ключевые слова: произвольные бимануальные движения, когнитивный стиль полезависимость-поленезависимость.

\section{PECULIARITIES OF LEVELS OF MOTION ACTIVITY AND CORRELATION RELATIONSHIPS OF CHARACTERISTICS OF BIMANUAL COORDINATION IN PERSONS WITH DIFFERENT TYPES OF COGNITIVE STYLE OF FIELD DEPENDENCE-FIELD INDEPENDENCE Sokolova N.I., Tkachenko P.V., Petrova E.V.}

Department of Normal Physiology named after Professor A.V. Zavyalov of Kursk State Medical University, Kursk

The levels of bimanual coordination in men and women related to different types of cognitive style of field dependencefield independence were studied by the method of support measurement. The comparison of average values of the coordination indices and their variability shows possible mechanisms of sex differences, connected to approaches to solving perceptual problems. The revealed peculiarities of correlation comparisons of characteristics of motion activity allowed to reveal the connections between a level of perception and a type of orientation with the features of bimanual movements' realization. The men's connections are differentiated according to time of task performance, those of women - due to implementation defect of a track and its complexity that confirms the differences in the methods of controlling the relevant activity. At the same time, women have different levels of workability in groups with polar types of cognitive style what is determined by the peculiarities of perception and consolidation of effector manifestations when a task becomes more complicated.

Keywords: arbitrary bimanual movement, cognitive style field dependence-field independence.

Известно, что эффективная реализация произвольных двигательных программ зависит от настройки и сонастройки сенсорных и эффекторных систем на различных уровнях ЦНС. В связи с этим остаются крайне актуальными вопросы профессионального отбора на производства и в спорте, требующие специализированной двигательной активности. При изучении бимануальной координации нами были описаны основные закономерности устойчивых внутрисенсорных и сенсорно-эффекторных взаимоотношений при выполнении сложноскоординированных движений руками $[4,6,7]$. Выявлены существенные половые различия в стратегиях выполнения манипуляторных действий, определяемые, в том числе, и выбором разных тактик выполнения заданий у мужчин и женщин [5].
Не вызывает сомнений, что познавательная деятельность и ее устойчивые процессуальные особенности, характеризующие своеобразие способов получения, переработки и воспроизведения, а также способов и стратегий контроля, будет влиять на уровень произвольной двигательной активности. Подобные особенности определяются как когнитивные стили, из которых, на наш взгляд, наиболее интересным представляется стиль полезависимостьполенезависимость, введенный в научный обиход Г. Уиткиным (1954), в связи с изучением соотношения в перцептивной деятельности зрительных и проприоцептивных ориентиров [2]. Естественно, что способ пространственной ориентации и деятельности связан со способностью структурировать пространство. По- 
этому поленезависимость рассматривается как умение преодолевать видимое поле и структурировать его, выделяя в нем отдельные элементы, а полезависимость - это качество познавательной деятельности, когда все элементы видимого поля жестко связаны и трудно отделимы от пространственного фона. Изучению указанного когнитивного стиля в различных сферах деятельности посвящено большое количество работ [2, 8, 9]. В то же время вопрос о влиянии принадлежности к тому или иному типу стиля на уровень пространственных произвольных целенаправленных движений рук остается практически неизученным с учетом обнаруженных особенностей бимануальной координации у испытуемых разных полов, что и послужило целью нашей работы.

\section{МАТЕРИАЛЫ И МЕТОДЫ ИССЛЕДОВАНИЯ}

В исследовании добровольно на основе информированного согласия приняли участие 42 мужчины (22 - полезависимые, 20 поленезависимые) и 43 женщины (22 полезависимые, 21 - поленезависимые) в возрасте от 18 до 20 лет. Уровень произвольной бимануальной двигательной активности оценивался методом суппортметрии. При выполнении каждого из шести заданий регистрировались общее время выполнения задания (ОВ), время на контуре задания (ВНК), время вне контура задания (ВВК) и количество ошибок (КО), на их основе рассчитывались скорость прохождения трека (СК), скорость реакции при исправлении ошибки (СР) и интегральный показатель координации (ИПК) [5].

Для определения когнитивного стиля полезависимость-поленезависимость (ПЗ-ПНЗ) использовалась методика включенных фигур (тест Готшильда). Методика реализовывалась посредством авторского программного обеспечения. После демонстрации трех вариантов с правильным выбором поочередно предъявлялись сложные фигуры. Фиксировались время поиска фигуры и количество ошибок. Индекс ПЗ-ПНЗ рассчитывался по формуле: количество правильных ответов/общее время в мин. При индексе $\geq 2,5$ испытуемый считается поленезависимым, а при индексе $\leq 2,5-$ полезависимым.

При статистической обработке рассчитывали средние значения (M) рассматриваемых характеристик с ошибкой (m), их коэффициенты вариации, определяли прямолинейные (r) взаимосвязи между изучаемыми показателями, рассчитывали коэффициент суммарной многосторонней скоррелированности $(\Sigma \mathrm{r})[1,3,5]$.

\section{РЕЗУЛЬТАТЫ ИССЛЕДОВАНИЯ И ИХ ОБСУЖДЕНИЕ}

При сравнении средних значений показателей бимануальной координации в группах испытуемых мужского пола, характеризующихся полярными когнитивными стилями (ПЗ-ПНЗ), статистически значимых различий выявлено не было. В аналогичных группах женщин выявлены достоверные различия между ОВ и ВНК шестого задания $(\mathrm{p}<0,05)$. При этом указанные характеристики координации движений выше в группе полезависимых испытуемых.

Сравнение показателей уровня координации между группами испытуемых мужского и женского пола, относящихся к полезависимому типу когнитивного стиля, показало, что общее время выполнения первого $(\mathrm{p}<0,05)$, второго $(\mathrm{p}<0,01)$ и шестого $(\mathrm{p}<0,05)$ заданий больше у полезависимых женщин. Аналогичная закономерная картина наблюдается и в отношении времени на контуре задания. Так, ВНК больше у женщин при прохождении трека первого ( $<<0,01)$, второго $(\mathrm{p}<0,01)$ и шестого $(\mathrm{p}<0,05)$ двигательных заданий. Что касается ВВК, то этот показатель также статистически значимо выше у испытуемых женского пола при прохождении шестого трека $(\mathrm{p}<0,05)$.

Анализ достоверности различий расчетных показателей суппортметрии, характеризующихся полезависимым стилем, показал, что скорость выполнения задания выше у испытуемых мужского пола. При этом статистически значимо при прохождении первого $(\mathrm{p}<0,01)$, второго $(\mathrm{p}<0,05)$ и шестого $(\mathrm{p}<0,05)$ треков. ИПК больше у мужчин при реализации только шестого двигательного задания $(\mathrm{p}<0,01)$.

В группах поленезависимого когнитивного стиля ВВК $(\mathrm{p}<0,01)$ и $\mathrm{KO}(\mathrm{p}<0,05)$ достоверно больше у женщин и только при выполнении второго двигательного задания. Интегральный показатель координации при реализации второго $(\mathrm{p}<0,05)$ и третьего $(\mathrm{p}<0,05)$ треков выше у испытуемых мужского пола.

Сравнение вариативности показателей бимануальной координации у мужчин с полярными типами рассматриваемого когнитивного стиля показало, что коэффициенты вариации части характеристик у поленезависимых испытуемых больше, чем у полезависимых. Так, достоверно отличаются ОВ первого ( $<<0,05)$, второго $(\mathrm{p}<0,05)$, четвертого $(\mathrm{p}<0,05)$, пятого $(\mathrm{p}<0,05)$ и шестого $(\mathrm{p}<0,01)$ двигательных заданий. Аналогичная картина наблюдается и в отношении времени на контуре задания. В отношении показателей дефекта реализации двигательных задач (ВВК и КО) отмечены их статистически значимые различия при прохождении пятого и шестого треков 
$(\mathrm{p}<0,05)$. Вариативность скорости реализации произвольных координаций, так же как и временные характеристики, достоверно больше в группе поленезависимых мужчин при выполнении первого $(\mathrm{p}<0,05)$, второго $(\mathrm{p}<0,05)$, четвертого $(\mathrm{p}<0,05)$, пятого $(\mathrm{p}<0,05)$ и шестого $(\mathrm{p}<0,01)$ заданий. Коэффициент вариации СР статистически больше только при прохождении первого трека. Что касается вариативности ИПК, то различия схожи с выявленными у показателей дефекта реализации заданий, т.е. различаются при выполнении пятого $(\mathrm{p}<0,05)$ и шестого $(\mathrm{p}<0,01)$ заданий.

Анализ коэффициентов вариации характеристик суппортметрии в полярных группах испытуемых женского пола показал менее выраженные различия по сравнению с мужчинами. Так, статистически значимо отличаются ОВ при реализации первого трека $(\mathrm{p}<0,05)$, ВНК при прохождении второго $(\mathrm{p}<0,001)$ и шестого заданий $(\mathrm{p}<0,01)$. При этом вариативность указанных характеристик больше у женщин, характеризующихся полезависимым стилем. В то же время коэффициенты вариации ВВК второго и четвертого заданий больше в группе поленезависимых $(\mathrm{p}<0,05)$, как и вариативность КО первого и второго треков $(\mathrm{p}<0,05)$, а также СР и ИПК второго задания $(\mathrm{p}<0,05)$.

Существенные различия выявлены при сравнении коэффициентов вариации показателей уровня координации сочетанных движений рук в группах мужчин и женщин, характеризующихся полезависимостью. При этом во всех случаях вариативность рассматриваемых характеристик выше у испытуемых женского пола. Так, для ОВ при реализации первого $(\mathrm{p}<0,01)$, второго $(\mathrm{p}<0,01)$, четвертого $(\mathrm{p}<0,05)$, пятого $(\mathrm{p}<0,05)$ и шестого $(\mathrm{p}<0,001)$ треков. Аналогичная картина выявлена и для ВНК первого $(\mathrm{p}<0,05)$, второго $(\mathrm{p}<0,001)$, четвертого $(\mathrm{p}<0,05)$, пятого $(\mathrm{p}<0,05)$ и шестого $(\mathrm{p}<0,001)$ заданий. Вариативность ВВК достоверно отличается только при выполнении третьего двигательного задания $(\mathrm{p}<0,05)$, как и КО $(\mathrm{p}<0,05)$. Кроме того коэффициент вариации КО больше и при прохождении пятого трека $(\mathrm{p}<0,05)$. Скорость выполнения бимануальных движений характеризуется большей вариативностью при выполнении всех заданий кроме третьего ( $<<0,05-\mathrm{p}<0,01)$, а для СР только при реализации первого трека $(\mathrm{p}<0,05)$. Вариативность ИПК статистически значимо отличается при прохождении второго-шестого контуров $(\mathrm{p}<0,05-\mathrm{p}<0,001)$.

В то же время вариативность рассматриваемых показателей суппортметрии в группах испытуемых мужского и женского пола, относящихся к поленезависимому стилю, выражена в меньшей степени с преобладанием значений у женщин.
Установлены достоверные

различия

коэффициентов вариации ВВК при выполнении четвертого задания $(\mathrm{p}<0,05), \mathrm{CP}$ третьего трека $(\mathrm{p}<0,05)$, а также ИПК при решении второй и третьей двигательных задач $(\mathrm{p}<0,01)$.

Теперь рассмотрим особенности межсистемных и внутрисистемных корреляционных взаимоотношений показателей бимануальной координации у испытуемых разных полов, относящихся к полярным когнитивным стилям.

Так, при рассмотрении межсистемных прямолинейных корреляционных взаимоотношений значения коэффициента, определяющего когнитивный тип, с показателями суппотметрии у поленезависимых мужчин установлено, что суммарная скоррелированность составила $\Sigma \mathrm{r}=4,81$ за счет достоверных отрицательных корреляционных связей с ОВ третьего и шестого заданий и с ВНК шестого задания. Кроме того, выявлены положительные корреляционные связи с СК и ИПК пятого и шестого треков. Суммарная многосторонняя скоррелированность значения коэффициента, определяющего полезависимость испытуемых мужского пола, составила значение $\Sigma \mathrm{r}=13,27$. Такой более высокий уровень межсистемных многосторонних взаимоотношений определяется положительными прямолинейными связями с ОВ и ВНК первого, второго, четвертого, пятого и шестого двигательных заданий. А также отрицательными корреляциями с СК всех заданий, с СР третьего и шестого треков, интегральным показателем координации первого, третьего, четвертого, пятого и шестого контуров.

У испытуемых женского пола коэффициент поленезависимости с показателями суппортметрии не коррелирует. Что касается группы полезависимых, то здесь суммарная межсистемная многосторонняя прямолинейная скоррелированость находится на уровне $\Sigma \mathrm{r}=5,29$. Эта сумма формируется из отрицательных связей коэффициента с ОВ, ВНК и ВВК первого и второго заданий и КО второго задания. Кроме того, выявлены положительные прямолинейные связи с СК и ИПК первого и второго двигательных заданий.

Рассмотрение особенностей внутрисистемных прямолинейных корреляционных связей показателей суппортметрии у мужчин как полезависимого, так и поленезависимого типа (табл. 1) показало однотипность ранжирования характеристик уровня координации вне зависимости от вида двигательного задания. Так, первые ранги, достоверно между собой не различаясь ( $>0,05)$, занимают временные характеристики ОВ и ВНК и определяемые ими скорость выполнения задания и ИПК. Последние же ранги занимают показатели 
дефекта реализации двигательных проб ВВК, КО и СР.

В группе испытуемых женского пола, относящихся к полярным типам когнитивного стиля (табл. 1), внутрисистемная многосторонняя скоррелированность показателей суппортметрии в целом схожа с таковой у мужчин. Однако наблюдается смещение ИПК на первые места ранжированных рядов, за которым следуют временные характеристики координации и СК, статистически значимо не различаясь друг с другом. Последние же позиции остаются за характеристиками, отражающими неэффективность бимануальной координации.

Сравнивая уровень многосторонней скоррелированности показателей координации у мужчин, относящихся к полярным типам полезависимости-поленезависимости (рис. 1), установлено, что достоверно выше уровень взаимосвязей характеристик выполнения первого задания у поленезависимых испытуемых по сравнению с полезависмыми. Во всех остальных сравнениях статистически значимых различий не выявлено.

Таблица 1

Суммарная многосторонняя скоррелированность ( $(\mathrm{r})$ показателей бимануальной координации

\begin{tabular}{|c|c|c|c|c|c|c|c|}
\hline \multicolumn{8}{|c|}{ Мужчины } \\
\hline \multicolumn{8}{|c|}{ Полезависимые } \\
\hline Показатель & OB & BHK & BBK & КO & CK & $\mathrm{CP}$ & ИПК \\
\hline$(\Sigma \mathrm{r})$ & 16,28 & 15,09 & 7,54 & 7,03 & 16,07 & 0 & 16,18 \\
\hline Ранг & 1 & 4 & 5 & 6 & 3 & 7 & 2 \\
\hline \multicolumn{8}{|c|}{ Поленезависимые } \\
\hline$(\Sigma r)$ & 17,58 & 17,7 & 6,01 & 5,38 & 15,89 & 4,35 & 17,62 \\
\hline Ранг & 3 & 1 & 5 & 6 & 4 & 7 & 2 \\
\hline \multicolumn{8}{|c|}{ Женщины } \\
\hline \multicolumn{8}{|c|}{ Полезависимые } \\
\hline$(\Sigma r)$ & 15,5 & 13,52 & 9,64 & 7,67 & 16,89 & 2,85 & 20,38 \\
\hline Ранг & 3 & 4 & 5 & 6 & 2 & 7 & 1 \\
\hline \multicolumn{8}{|c|}{ Поленезависимые } \\
\hline$(\Sigma \mathrm{r})$ & 20,67 & 17,18 & 14,46 & 11,89 & 17,66 & 3,3 & 21,39 \\
\hline Ранг & 2 & 4 & 5 & 6 & 3 & 7 & 1 \\
\hline
\end{tabular}

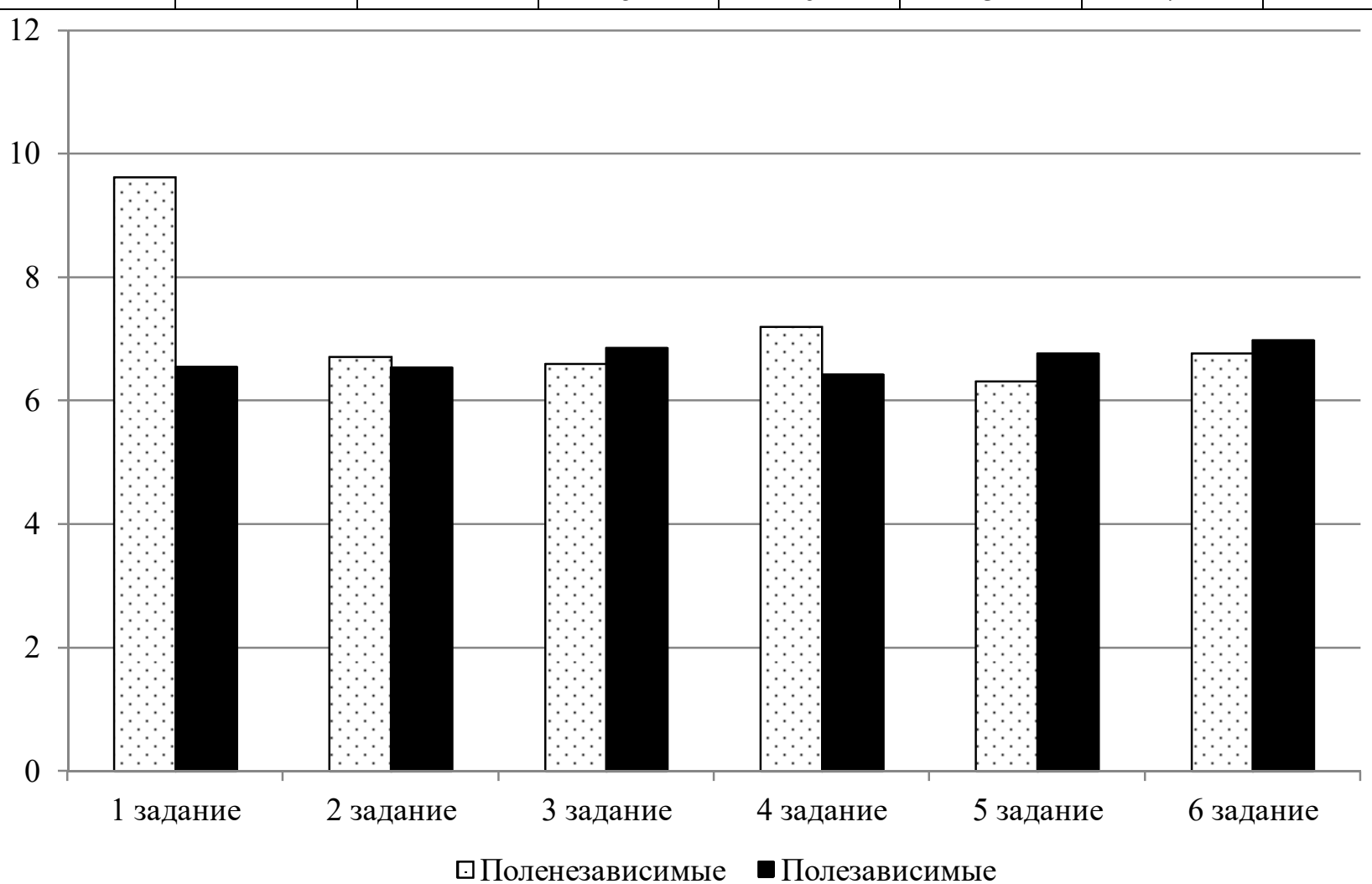

Рис. 1. Суммарная многосторонняя скоррелированность $(\Sigma \mathrm{r})$ показателей суппортметрии по каждому заданию у мужчин. 


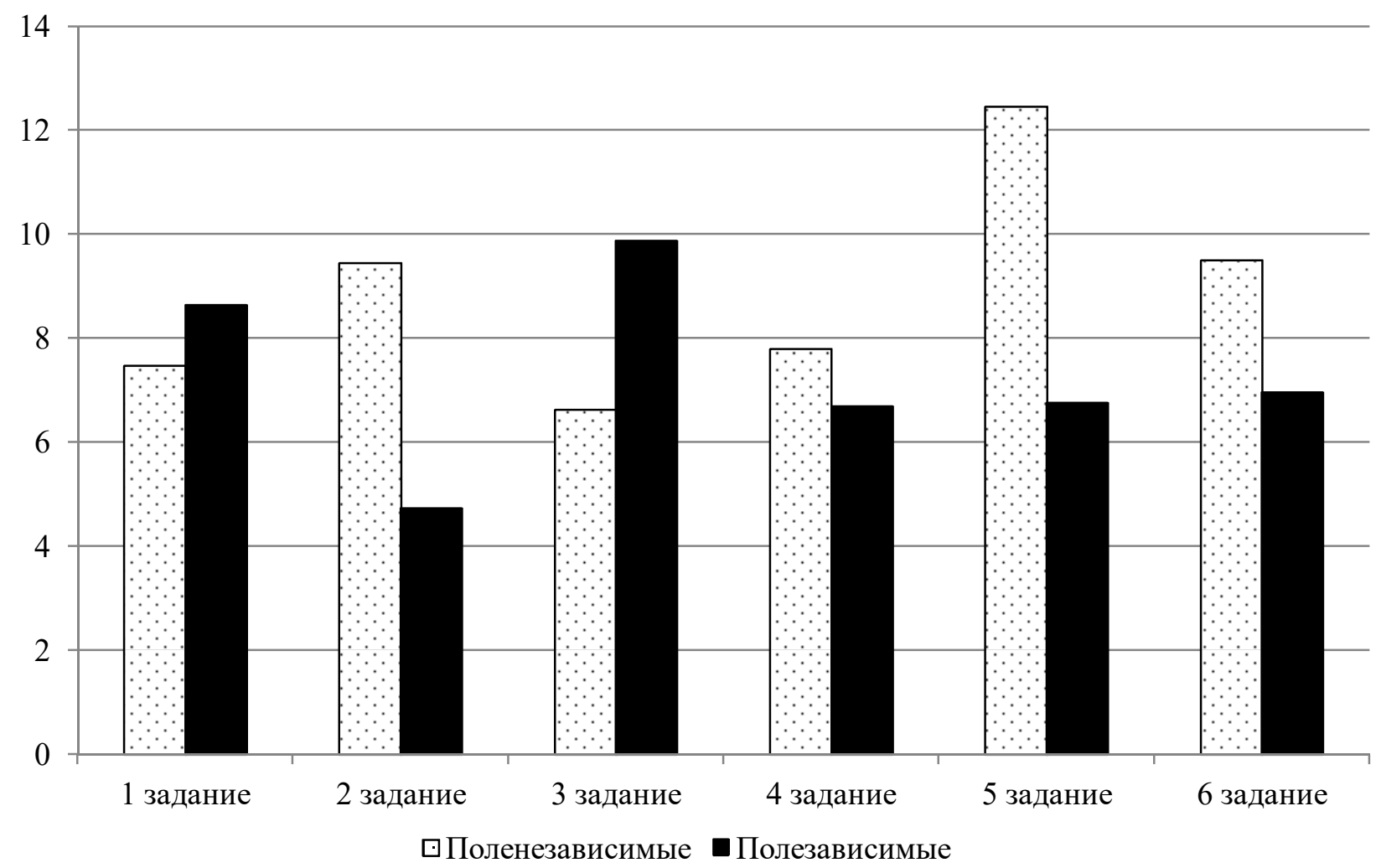

Рис. 2. Суммарная многосторонняя скоррелированность $(\Sigma r)$ показателей суппортметрии по каждому заданию у женщин.

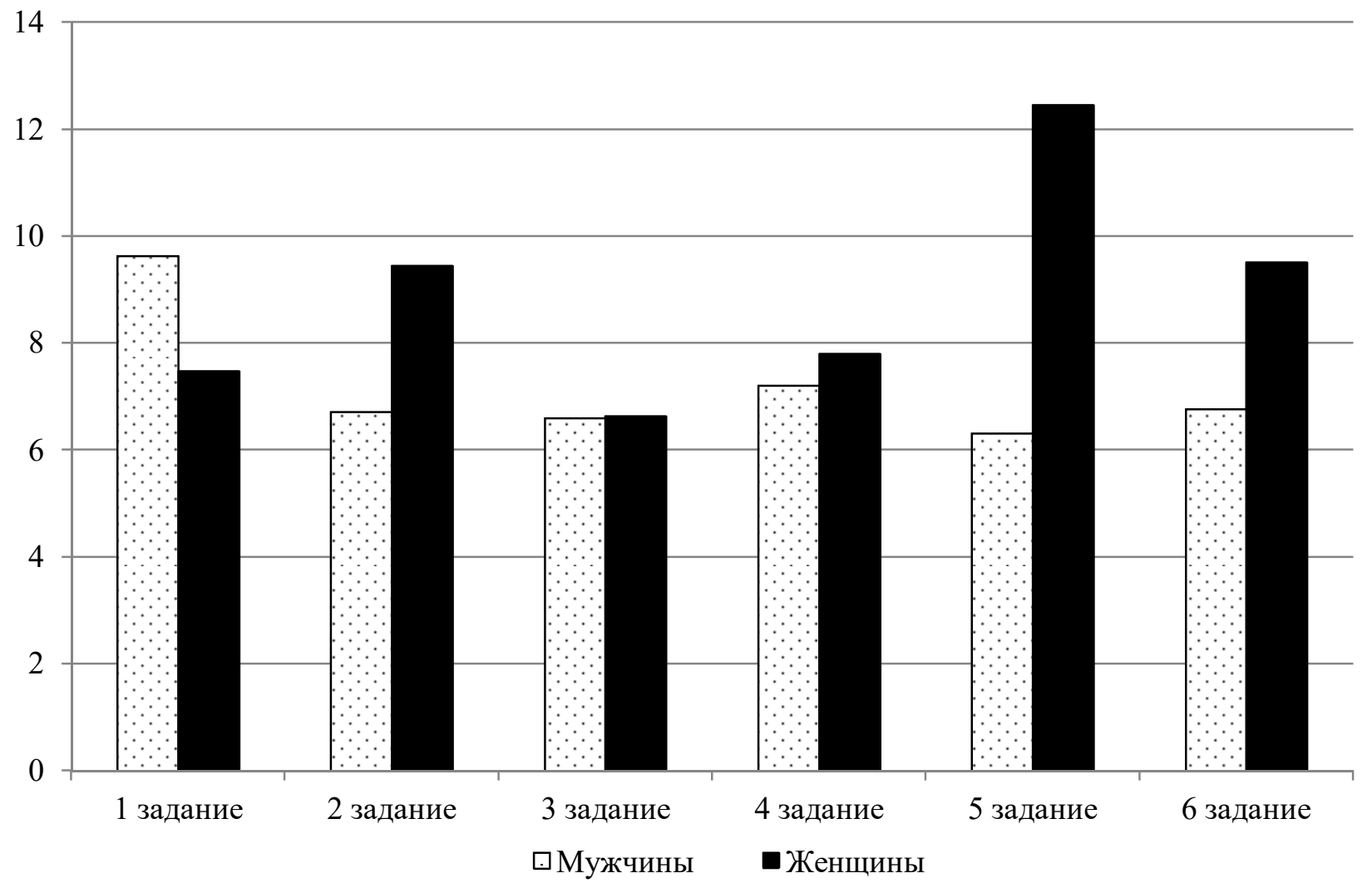

Рис. 3. Суммарная многосторонняя скоррелированность $(\Sigma r)$ показателей суппортметии по каждому заданию у испытуемых поленезависимого типа. 


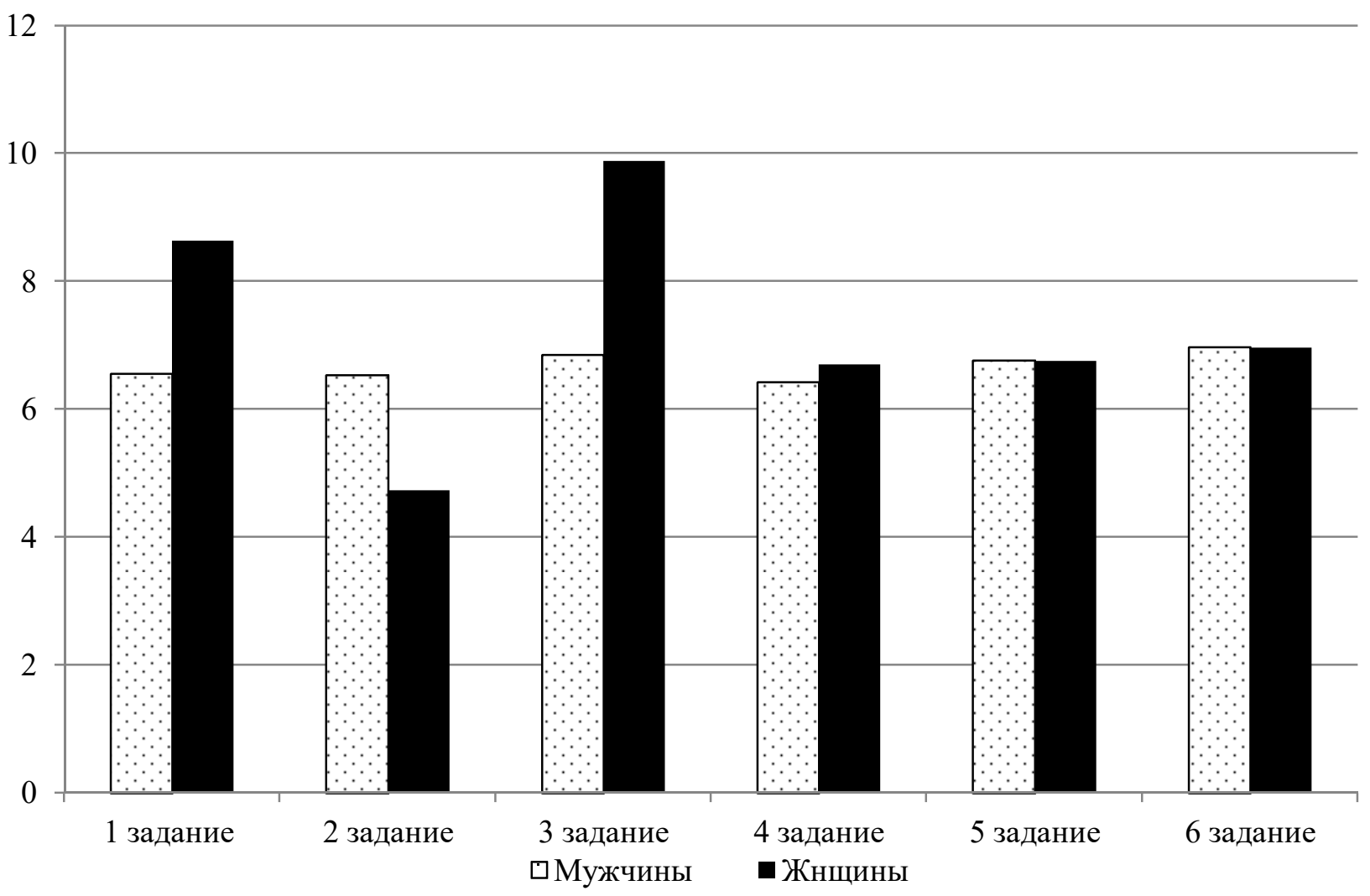

Рис. 4. Суммарная многосторонняя скоррелированность $(\Sigma r)$ показателей суппортметии по каждому заданию у испытуемых полезависимого типа.

Аналогичное сопоставление у женщин (рис. 2) показало, что у поленезависимых испытуемых теснота прямолинейных связей достоверно более выражена при реализации треков второго, пятого и шестого заданий. В то же время показатели, характеризующие прохождение контура третьего задания, более взаимосвязаны у поленезависимых женщин. Суммарная многосторонняя прямолинейная корреляция показателей выполнения первого и четвертого заданий в рассматриваемых группах испытуемых достоверно не различается.

Анализируя уровень прямолинейных корреляционных взаимоотношений $\mathrm{y}$ испытуемых разных полов, относящихся к поленезависимому типу когнитивного стиля (рис. 3), установлено, что наибольший уровень взаимоотношений относится к характеристикам первого бимануального задания у мужчин. В то же время у женщин, по сравнению с мужчинами, наиболее взаимосвязаны показатели выполнения второго, пятого и шестого заданий, а при выполнении третьего и четвертого статистически значимых различий не выявлено.

У испытуемых противоположного полезависимого типа (рис. 4) характеристики выполнения последних трех заданий скоррелированы на одном уровне и у мужчин и у женщин. В то же время показатели выполнения первой и третьей координационных проб достоверно больше взаимосвязаны у женщин, а второго задания - у мужчин.

Рассмотренные различия характеристик бимануальной координации у испытуемых мужского и женского полов, относящихся к полярным типам когнитивного стиля, свидетельствуют о том, что у мужчин уровень координации не зависит от типовой принадлежности. В то же время у женщин, ориентирующихся на внешние источники информации, увеличивается время реализации последнего сложного задания, состоящего из множества разнообразных геометрических элементов.

Выявленные половые различия значений суппортметрии у мужчин и женщин полезависимого когнитивного стиля в целом подтверждают данные, полученные нами ранее $[4,7]$. Так, уровень пространственной координации существенно выше у испытуемых мужского пола, что и проявилось в значимых закономерных различиях временных и расчетных характеристик бимануальных движений. В то же время у испытуемых, в меньшей степени подверженных влиянию внешнего контекста, т.е. относящихся к поленезависимому типу, основные различия проявляются в характеристиках дефекта выполнения задания с превалированием значений у женщин. Обращает на себя внимание, что в рассматриваемых закономерностях выраженное 
значение имеет тип двигательного задания, поскольку основные различия относятся к показателям первого «запального» (тренировочного) задания, а также наиболее сложных - второго и шестого, что подтверждается рядом проведенных ранее исследований $[4,5,6,7]$.

Вариативность показателей суппорметрии, характеризующих уровень координации у мужчин, выше у поленезависимых. У женщин разнообразие выражено в меньшей степени, при этом коэффициенты вариации части временных маркеров выше у полезависимых испытуемых, а показателей дефекта в противоположной группе. Интересно, что рассмотрение половых различий выявило исключительное преобладание значений коэффициентов вариации у испытуемых женского пола вне зависимости от типа когнитивного стиля с достаточно четкой зависимостью от сложности двигательного задания. Однако половые различия вариативности у поленезависимых выражены в меньшей степени. При этом, вероятно, в структуру ИПК больший вклад вносят не временные характеристики координации, а ошибочные действия. Поскольку коэффициент вариации является неименованным показателем, он пригоден для сравнения разных признаков или одного и того же признака, но в группах с достоверно различной средней величиной, что и наблюдается при сравнении характеристик координации в группах мужчин и женщин с разными типами когнитивного стиля ПЗ-ПНЗ. Проведенное нами сопоставление коэффициентов вариации отражает не только индивидуальные и групповые различия, но и косвенно указывает на различные стратегии реализации двигательных координаций у мужчин и женщин с учетом типа когнитивного стиля $[3,5]$.

Результаты межсистемного корреляционного сопоставления между значениями коэффициента, определяющего принадлежность к тому или иному типу когнитивного стиля, и показателями координации подтверждают различия используемых стратегий как у испытуемых разных полов, так и относящихся к полярным типам. Во-первых, значения показателей суппортметрии более тесно связаны с уровнем полезависимости с выраженной теснотой связей у мужчин. Во-вторых, уровень этих взаимосвязей определяется противоположными, разнонаправленными соотношениями в парах сопоставлений. Уровень бимануальной координации у поленезависимых испытуемых в меньшей степени связан с соответствующим коэффициентом, что представляется вполне закономерным. При этом если у женщин корреляционных связей нами выявлено не было, то у мужчин коэффициенты корреляции имеют противоположный знак в одноименных сопоставлениях у испытуемых полезависимого типа.

Структура внутрисистемной многосторонней скоррелированности показателей суппортметрии в целом соответствует описанной нами ранее [5] с выраженной теснотой взаимосвязей временных характеристик координации и ИПК и низким уровнем взаимоотношений показателей дефекта вне зависимости от когнитивного типа и вида задания. При этом у женщин уровень суммарной корреляции выше, чем у мужчин.

В то же время достоверно наибольшим уровнем взаимоотношений характеризуются показатели координации при выполнении только первого тренировочного задания у поленезависимых мужчин. У женщин подобная закономерность наблюдается в отношении наиболее сложных заданий, что подтверждает высказанные нами предположения о разных уровнях настройки двигательной системы в зависимости от когнитивных особенностей, ориентации на внешние или внутренние представлений и сложности контура двигательного задания.

Таким образом, проведенное нами исследование показало, что уровень пространственной координации бимануальных движений соотносится с принадлежностью испытуемых к тому или иному типу когнитивного стиля полезависимость-поленезависимость. Выявленные половые различия произвольной двигательной активности, а также вариативности показателей суппортметрии подтверждают результаты наших предыдущих исследований и раскрывают возможные новые механизмы этих различий, связанные с особенностями решения перцептивных задач. Рассмотрение межсистемных корреляционных взаимоотношений подтвердило связь уровня восприятия и типа ориентации с уровнем произвольной двигательной активности. При этом у мужчин выявленные связи более выражены и дифференцированы по временным показателям координации, а у женщин - по показателям дефекта реализации трека и его сложности. Это подтверждает наличие различий в способах контроля соответствующей деятельности. Изучение внутрисистемных корреляционных связей показателей уровня координации движений рук выявило однотипность этих взаимоотношений с преобладанием скоррелированности ИПК. В то же время у женщин суммарный уровень этих взаимосвязей выше, чем у мужчин, вне зависимости от типа 
стиля. Сравнительных анализ тесноты связей показателей суппортметрии при выполнении заданий различной сложности выявил, что у поленезависимых мужчин возрастает уровень скоррелированности только при прохождении первого тренировочного трека, во всех остальных случаях показатели координации у полярных типов друг с другом не различаются. У женщин внутрисистемная скоррелированность дифференцирована по сложности задания и отражает разную степень врабатываемости у полезависимых и поленезависмых, т.е. определяется особенностями восприятия с консолидацией эффекторных проявлений при усложнении задачи. Это подтверждается и сравнением уровня взаимосвязей характеристик координации у испытуемых разных полов, относящихся к одному типу когнитивного стиля. В целом ориентация только на внешние источники информации приводит к снижению уровня произвольной двигательной активности ввиду зависимости от контекста и неэффективной коррекции произвольной программы без использования внутренних источников.

\section{ЛИТЕРАТУРА}

1. Завьялов А.В. Корреляция функций организма. М. : Медицина, 1990. - 246 с.

2. Ильин Е.П. Психология индивидуальных различий. - СПб. : Питер, 2011. - 701 с.
3. Плохинский Н.А. Биометрия. - М.: Изд-во МГУ, 1972. $-230 \mathrm{c}$

4. Ткаченко П.В., Бобынцев И.И. Взаимосвязь амплитудных характеристик стимуляционной миографии с показателями бимануальной координации у мужчин и женщин при освоении двигательных заданий различной сложности // Бюл. экспериментальной биологии и медицины. 2014. - Т. 158, № 12. - С. 669-672.

5. Ткаченко П.В., Бобынцеев И.И. Соотношение моторных и сенсорных функций человека. Курск : Изд-во КГМУ, 2014. - 264 с.

6. Ткаченко П.В. Бобынцев И.И. Особенности взаимоотношений характеристик латентностей M-ответа и F-волны с показателями бимануальной координации у мужчин и женщин при выполнении двигательных заданий различной сложности // Курский науч.-практич. вестн. «Человек и его здоровье». - 2015. - № 1. - С. 115-119.

7. Ткаченко П.В., Бобынцев И.И. Особенности соотношений характеристик эффекторного аппарата и показателей бимануальной координации // Курский науч.-практич. вестн. «Человек и его здоровье». - 2015. - № 3. - С. 126-132.

8. Шкуратова И.П. Когнитивный стиль и общение. Ростов н/Д. : Изд-во Ростовского пед. ун-та, 1994. $-156 \mathrm{c.}$

9. Шкуратова И.П. Габдулина Л.И. Полезависимость-поленезависимость как детерминанта индивидуально-стилевых особенностей педагогического общения // Психол. вестн. - 1998. - Вып. 3. C. 226-235. 